\title{
Editorial
}

\section{Hypertension Control in India: Lessons from Jaipur Heart Watch}

\author{
Rajeev Gupta \\ Editor, RUHS Journal of Health Sciences; Chair, Academic and Research Development Unit, Rajasthan University of \\ Health Sciences, Jaipur, Rajasthan, India \\ DOI:10.37821/ruhsjhs.6.1.2021.374
}

Forty years ago, we set upon a journey on cardiovascular disease epidemiology and prevention of ischemic heart disease (IHD). ${ }^{1}$ The initial focus was on estimation of burden of IHD in urban and rural medical clinics in Rajasthan and importance of conventional risk factors using secondary prevention data. We identified that smoking, hypertension, and high cholesterol were important risk factors. $^{2-4}$ To determine burden of IHD risk factors in rural and urban populations in the state we initiated a series of epidemiological studies under the banner of Jaipur Heart Watch (JHW) in early 1990's. ${ }^{1}$ That hypertension or high blood pressure (BP) is a major cardiovascular risk factor has been well known for decades. ${ }^{5}$ Our studies also reported high burden of this condition in both rural and urban locations in the state of Rajasthan. ${ }^{6}$ We also documented that hypertension was increasing in India and the current nationwide studies have reported that it is present in $20-24 \%$ urban and $15-20 \%$ rural populations. ${ }^{7,8}$ Indeed, current data show that hypertension is the most important risk factor for disease burden and mortality in the country. ${ }^{9}$ Prospective studies especially the Prospective Urban Rural Epidemiology (PURE) study, in which we are one of the collaborators, has reported that hypertension is the most important cardiovascular risk factor globally as well as in our region. ${ }^{10}$

Status of hypertension control: The most important issue in prevention of hypertension-related diseases (IHD, strokes, ocular, and renal disease, etc.) is control of blood pressure. ${ }^{5}$ Target BP of less than 140/90 $\mathrm{mmHg}$ has been recommended for reducing risk by various international guidelines, although the recent American guidelines recommend a target of $<130 / 85 \mathrm{mmHg}$ for cardiovascular disease prevention. ${ }^{11}$ In the present focus issue on hypertension, Gautam shares the journey of evolution of hypertension guidelines and BP control targets over the past century. ${ }^{12}$ In most cases, hypertension control to tar- gets can be achieved using a combination of lifestyle modifications (weight loss, salt restriction, healthy diet intake, exercise, and regular physical activity, and avoidance of smoking and alcohol) along with a number of inexpensive widely available medicines. ${ }^{5}$ Then why is the control of BP low globally and still lower in India and other developing countries. ${ }^{13}$ I believe that social determinants of health- poverty, illiteracy, lack of universal healthcare, status of health financing, low affordability and low availability are important. A multitude of social factors specific to India are important and have been highlighted in one of our previous studies, ${ }^{14}$ and in an editorial in Lancet some months ago. ${ }^{13}$

In JHW studies, we evaluated the status of hypertension awareness, treatment, and control over a 25 -year period using serial cross sectional studies also known an interrupted time-series studies from the years 1991-1992 to 2015-2017. . $^{15} \mathrm{JHW}-1$ urban and rural studies conducted in early 1990's reported a very low awareness, treatment, and control status in those with hypertension and known hypertension. The situation has significantly improved since then and subsequent studies have reported increasing awareness, treatment, and control (Figure). Data from the more recent studies (JHW-6 and upcoming JHW-7) have reported that hypertension awareness in an urban Indian population is currently about $50-60 \%$, treatment in 30 $40 \%$, and control in $20-25 \%$. Despite the increase, the situation in hypertension control is still below par and much lower than studies in most developed countries where awareness is in more than $80 \%$ and control in about $50-70 \%{ }^{16}$ A few developing countries in Africa and South America have better control rates than us. ${ }^{17}$

Strategies for better hypertension control: A number of strategies are available to achieve better BP control. ${ }^{18}$ These include improving social determinants of health, 


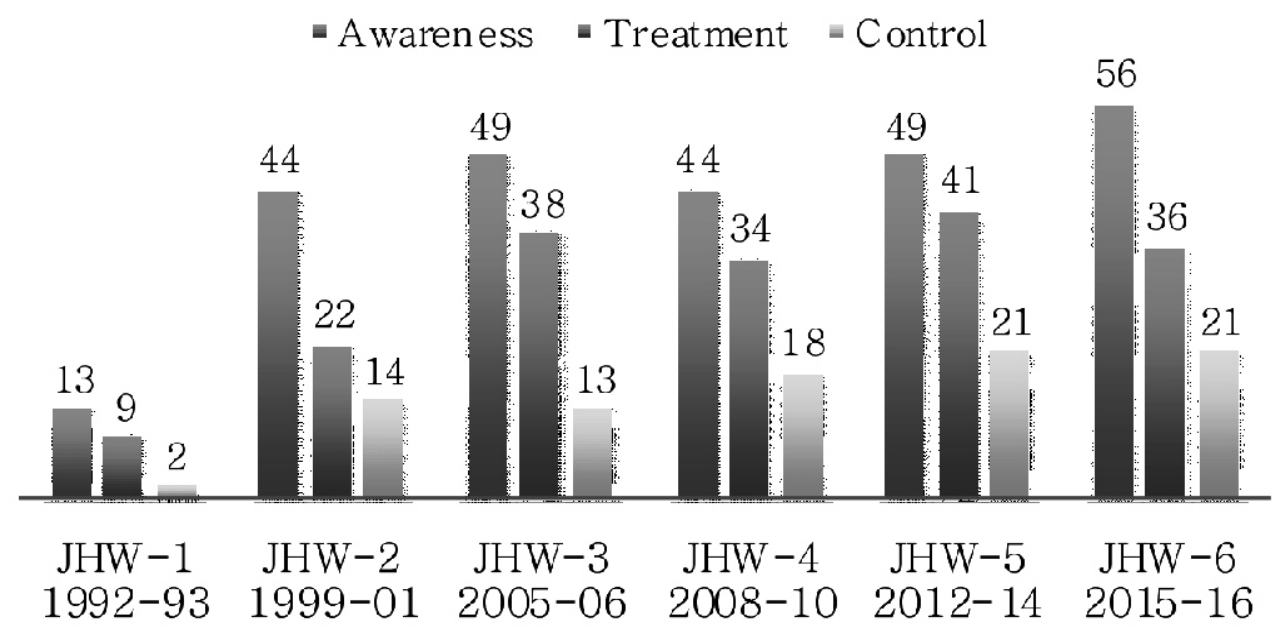

Figure: 25 year trends in hypertension awareness, treatment and control in Jaipur Heart Watch (JHW) Studies.

universal healthcare, better primary and secondary level care, better education of physician and non-physician health workers availability and affordability of highquality low-cost medicines, and better compliance and adherence to them by patients.

World Health Organization and United Nations' Sustainable Development Goals have identified important social determinants in context of hypertension. ${ }^{19}$ These include quality universal education, elimination of poverty, zero hunger, decent work and economic growth, reducing inequalities, sustainable communities and cities, responsible consumption and production, climate action, and fostering partnerships for the goals. Most countries, including India are signatories to this. Better quality universal healthcare and availability and affordability of healthcare are also important. The US Surgeon General has recommended three goals to support improvements in hypertension control: (a) make hypertension control a national priority; (b) ensure that the places where people live, learn, work, and play support hypertension control; and (c) optimize patient care for hypertension control..$^{20}$ More focus on all these factors is needed in India.

A comprehensive hypertension management and treatment protocol should be developed in India similar to most developed countries. The US Surgeon General has provided useful guidance in this regard. ${ }^{20}$ The protocol should focus on accurate BP measurement, setting optimal BP targets, overall cardiovascular risk assessment, teambased care approach, focus on lifestyle modification, guidance for medication initiation and intensification, consideration for medicine costs, supportive strategies for promoting medication adherence including technological innovations, and regular follow-up. Comprehensive cardiovascular risk reduction is crucial to achieve morbidity and mortality related to hypertension. Only then the hypertension control would be optimal.

\section{REFERENCES}

1. Gupta R. My journeys into cardiovascular epidemiology research in India. South Asian J Prev Cardiol. 2017; 21:4659.

2. Gupta R, Gupta KD, Sharma S, Gupta VP. Influence of cessation of smoking on long term survival in patients with coronary heart disease. Indian Heart J. 1993; 45:125-29.

3. Gupta R, Gupta KD, Kasliwal P. Influence of mild to moderate treated hypertension on 9 to 11 year mortality in patients with pre-existing coronary heart disease. $J$ Hum Hypertens. 1992; 6:313-16.

4. Gupta R, Gupta KD. Total cholesterol and mortality in patients with pre-existing coronary artery disease. Natl Med JIndia. 1992; 5:111-14.

5. Kotchen TA. Hypertensive vascular disease. In: Harrison's Principles of Internal Medicine. $20^{\text {th }}$ Ed. New York, Mc Graw-Hill. 2020;1890-906.

6. Gupta R, Gupta VP. Hypertension epidemiology in India: Lessons from Jaipur Heart Watch Studies. Curr Science 2009; 97:349-55.

7. Gupta R, Al-Odat NA, Gupta VP. Hypertension epidemiology in India. Meta-analysis of fifty-year prevalence rates and blood pressure trends. J Hum Hypertens. 1996; 10:465-72. 
hypertension epidemiology in India. J Hum Hypertens. 2019; 33:575-87.

9. Gupta R, Xavier D. Hypertension: The most important noncommunicable disease risk factor in India. Indian Heart $J$. 2018; 70:565-72.

10. Yusuf S, Joseph P, Rangarajan S, Islam S, Mente A, Hystad P et al. Modifiable risk factors, cardiovascular disease, and mortality in 155,722 individuals from 21 high-income, middle-income, and low-income countries (PURE): A prospective cohort study. Lancet. 2020 395;795-808.

11. Wander GS, Ram CVS. Optimal blood pressure goals recommended by the latest hypertension guidelines: India may benefit the most. Eur Heart J. 2018; 39:3012-16.

12. Gautam P. Comparative evaluation of major hypertension guidelines and practical implications. RUHS $J$ Health Sciences. 2021; 6:EPub.

13. Chow CK, Gupta R. Blood pressure control: A challenge to global health systems. Lancet 2019; 394: 613-15.

14. Gupta S, Dhamija JP, Mohan I, Gupta R. Qualitative study of barriers to adherence for anti-hypertensive medications among rural women in India. Int J Hypertens. 2019; 2019: 5749648.

15. Gupta R, Gupta VP, Prakash H, Agrawal A, Sharma KK, Deedwania PC. 25-year trends in hypertension prevalence, awareness, treatment and control in an urban population in India. Indian Heart J. 2018; 64: 802-07.

16. NCD Risk Factor Collaboration (NCD-RiSC). Long term and recent trends in hypertension awareness, treatment and control in 12 high-income countries: An analysis of 123 nationally representative surveys. Lancet. 2019;394:639-51.

17. Geldsetzer P, Manne-Goehler J, Marcus M-E, Ibert C, Zhumadilov Z, Wesseh CS et al. The state of hypertension care in 44 low-income and middle-income countries: a cross sectional study of nationally representa-tive individual level data from 1.1 million adults. Lancet. 2019; 394:652-62.

18. Gupta R, Khedar RS, Panwar RB. Strategies for better hypertension control in India and other lower-middle income countries. J Assoc Physicians India. 2016; 64:58-64.

19. Gupta R, Wood DA. Primary prevention of ischemic heart disease: populations, individuals and healthcare professionals. Lancet. 2019; 394:685-96.

20. US Department of Health and Human Services. The Surgeon General's call to action to control hypertension. Washington. Office of the Surgeon general. 2020.

\section{Corresponding Author}

Dr Rajeev Gupta, Department of Medicine, Eternal Heart Care Centre and Research Institute, Jagatpura Road, Jawahar Circle, Jaipur, India. PIN-302017. 